American Journal of Infectious Diseases 5 (3): 188-192, 2009

ISSN 1553-6203

(C) 2009 Science Publications

\title{
Probiotics the Good Neighbor: Guarding the Gut Mucosal Barrier
}

\author{
R.K. Rao, D.B. Polk, A. Seth and F. Yan \\ Department of Physiology, \\ University of Tennessee Health Science Center, Memphis TN, USA
}

\begin{abstract}
Problem statement: The disruption of gut barrier function plays a crucial role in the pathogenesis of not only gastrointestinal diseases, but also the diseases of liver and other organs. Mucosal protective factors that preserve the gut barrier integrity are beneficial in the prevention and treatment of such diseases. Probiotics is a group of helpful bacteria that protect the gastrointestinal mucosa from a variety of insults. Therefore, understanding the mechanism of probiotic-mediated protection of gut barrier function is an important area of investigation. Approach: Several studies had addressed the role of probiotics in the protection of gut barrier integrity. In a recent study, we investigated the role of Lactobacillus rhamnosus GG and two soluble proteins, p40 and p75, in the protection of gut barrier function in Caco-2 cell monolayer, a model of the intestinal epithelium. Results: Studies demonstrated that live or dead Lactobacillus rhamnosus GG prevents oxidative stress-induced disruption of tight junctions and barrier function in Caco-2 cell monolayers. The isolated soluble proteins of this probiotic, $\mathrm{p} 40$ and $\mathrm{p} 75$, also prevent hydrogen peroxide-induced tight junction disruption. This protective effect of probiotic proteins was mediated by the activation of ERK1/2 and protein kinase C isoforms, PKC $\beta$ I and PKCE. Conclusion: Lactobacillus rhamnosus GG prevent oxidative stress-induced disruption of intestinal epithelial tight junctions and barrier function, suggesting that preservation of epithelial barrier function is one of the mechanisms involved in the mucosal protective role of probiotics in the gut.
\end{abstract}

Key words: Intestine, mucosal protection, barrier junction, tight junction, occludin, ZO-1, protein kinase $\mathrm{C}, \mathrm{LGG}$, probiotics

\section{INTRODUCTION}

Probiotics, the live microorganisms that inhabit the gastrointestinal tract, confer health benefits to the host, such as nutritional assistance, maturation of immune system, protection of mucosal barrier function and prevention of injurious effects caused by xenobiotics and pathogens ${ }^{[1]}$. A flurry of basic and clinical studies on probiotics during the past decade lead $\mathrm{FAO} / \mathrm{WHO}$ to define probotics as "live microorganisms which, when consumed in adequate amounts as part of food, confer a health benefit on the host" ${ }^{\text {[2] }}$. Probiotics are considered to offer potential therapeutic applications in the prevention and treatment of different diseases. The current clinical applications of probiotics include treatment and/or prevention of inflammatory bowel diseases, diarrhea, irritable bowel syndrome, gluten intolerance, gastroenteritis and Helicobacter pylori infection ${ }^{[3]}$. A significant body of evidence indicates that probiotics regulate intestinal epithelial homeostasis, such as promotion of cell survival and barrier function $^{[4-6]}$, improve intestinal microbial ecology and regulate immune function ${ }^{[7]}$.

Lactobacillus rhamnosus: Probiotics in the gut include 30 species of Bifidobacteria, 52 species of Lactobacillus and others such as Saccharomyces, Streptococcus and Enterococcus. Lactobacillus rhamnosus GG (LGG), originally isolated from the healthy human intestine ${ }^{[8]}$, is one of the best-studied probiotic bacteria in clinical trials for treating and/or preventing several intestinal disorders, including inflammatory bowel diseases and diarrhea $^{[7]}$. Recent mechanistic studies using LGG as a model of probiotic bacterium find that LGG prevents cytokine-induced apoptosis in the intestinal epithelial cells through activation of Akt and prevention of p38MAPK activation ${ }^{[5]}$. More importantly, constituents recovered from LGG culture broth supernatant stimulate Akt action to prevent cytokine-induced apoptosis in intestinal epithelial cells ${ }^{[5]}$. Furthermore, two LGG-produced soluble proteins, $\mathrm{p} 75$ and $\mathrm{p} 40$, have been successfully purified and cloned. Both p75 and

Corresponding Author: R.K. Rao, Department of Physiology, University of Tennessee Health Science Center, Memphis TN, USA 
p40 activate Akt and regulate intestinal epithelial cell anti-apoptotic responses ${ }^{[6]}$. In the gastrointestinal tract LGG promote good digestion, boost immune system, increase resistance to infection and inhibit growth of pathogenic bacteria. One of the important mechanisms by which LGG exerts its beneficial effect in gut appears to be the protection of mucosal barrier function.

Tight junctions and gut barrier function: Gut mucosal barrier function prevents the diffusion of potential injurious factors from the gastrointestinal lumen into the tissue and eventually into the systemic circulation ${ }^{[9]}$. Disruption of barrier function and elevated permeability to luminal toxins, allergens and pathogens play a crucial role in the pathogenesis of a number of gastrointestinal diseases such as inflammatory bowel disease, celiac disease and alcoholic liver disease. A major component of the mucosa barrier function is the specialized intercellular junctional complex called tight junctions, a circumferential ring located at the apical end of the lateral membrane of the epithelial cells. Tight junctions are formed by the organization of a number of specific proteins such as occludin, zonula occludens (ZO-1, ZO2 and ZO-3), claudins and junctional adhesion molecule ${ }^{[9]}$. The assembly and disassembly of tight junctions are regulated by numerous intracellular signaling molecules ${ }^{[10]}$.

Proinflammatory factors such as reactive oxygen species $^{[11-15]}$, cytokines ${ }^{[16,17]}$ and toxins ${ }^{[18]}$ disrupt the tight junction and compromise the barrier function of the intestinal epithelium. Previous studies have demonstrated that hydrogen peroxide disrupts tight junctions in the Caco-2 cell monolayer by a mechanism involving phosphotidylinositol 3-kinase ${ }^{[15]}$ and c-Src ${ }^{[11]}$. Hydrogen peroxide induces a redistribution of tight junction and adherens junction proteins, occludin, ZO1 , E-cadherin and $\beta$-catenin, from the intercellular junctions into the intracellular compartments. The factors that prevent the inflammation-mediated disruption of the tight junction and barrier function may provide potential therapeutic benefit in the treatment of many gastrointestinal diseases.

Approach and results: LGG has been shown to protect the intestinal mucosal integrity by preventing the cytokine-induced apoptosis of epithelial cells ${ }^{[5]}$. The probiotic, Escherichia coli Nissle 1917, mediates up regulation of tight junction protein, $\mathrm{ZO}-1$ and prevents gut barrier disruption induced by sodium dextran sulfate-induced colitis ${ }^{[19]}$. In a recent study we evaluated the effect of LGG and proteins secreted by LGG (p75 and p40) on hydrogen peroxide-induced disruption of tight junctions in Caco-2 cell monolayers, a cell culture model of the intestinal epithelium ${ }^{[20]}$. Pretreatment of cell monolayers with p40 or p75 attenuated the hydrogen peroxide-induced decrease in TER and increase in inulin permeability in a time and dose-dependent manner. Probiotic proteins, p40 and p75 prevented hydrogen peroxide-induced redistribution of occludin, ZO-1, E-cadherin and $\beta$ catenin from the intercellular junctions and their dissociation from the detergent-insoluble fractions. Both p40 and p75 induced a rapid increase in the membrane translocation of PKC $\beta \mathrm{I}$ and PKCE. The attenuation of hydrogen peroxide-induced inulin permeability and redistribution of tight junction proteins by $\mathrm{p} 40$ and p75 was abrogated by Ro-32-0432, a PKC inhibitor. LGG proteins, p40 and p75 also rapidly increased the levels of phospho-ERK1/2 in the detergent-insoluble fractions. U0126 (a MAP kinase inhibitor) attenuated the $\mathrm{p} 40$ and p75-mediated reduction of hydrogen peroxide-induced tight junction disruption and inulin permeability.

The attenuation of hydrogen peroxide-induced increase in inulin permeability in Caco-2 cell monolayers by live or dead LGG suggested that certain component of LGG is responsible for this protective response. Protection of tight junctions from hydrogen peroxide by LGG supernatant and the purified proteins, $\mathrm{p} 40$ and $\mathrm{p} 75$, indicated that the proteins secreted by LGG are involved in the amelioration of oxidative stress-induced increase in paracellular permeability. A previous study demonstrated that the probiotics protect the intestinal epithelial cells from TNFo-induced apoptosis ${ }^{[5]}$. Although ZO-1 and occludin levels in detergent-insoluble fractions were dramatically reduced by $\mathrm{H}_{2} \mathrm{O}_{2}$ treatment, confocal microscopy showed that redistribution of occludin from the junctions was much more pronounced compared to the redistribution of $\mathrm{ZO}-$ 1 . This may be explained by proposing a possibility that $\mathrm{H}_{2} \mathrm{O}_{2}$ had only minimal effect on the interaction between $\mathrm{ZO}-1$ and claudins and therefore a significant portion of ZO-1 remains to be localized at the intercellular junctions. The adherens junction lies beneath the tight junction and is formed by the organization of E-cadherin and $\beta$-catenin. Evidence suggests that the adherens junction indirectly regulates the integrity of tight junction. The probiotic proteins (p40 and p75) also prevent hydrogen peroxide-induced redistribution of $\mathrm{E}$ - 
cadherin and $\beta$-catenin. Therefore, probiotics may stabilize both tight junction and adherens junction and preserve the integrity of barrier function.

Probiotic secretory proteins, LGG supernatant, p40 and p75, did not change the level of hydrogen peroxide, indicating that the effect of probiotic was not mediated by an antioxidant effect. This was further supported by the observation that LGG supernatant effectively prevented hydrogen peroxide-induced disruption of barrier function when the cells were pretreated with LGG supernatant and removed before the administration of hydrogen peroxide. P40 and p75 were effective in preventing hydrogen peroxide-induced disruption of tight junction even when they were administered only to the apical surface or the basal surface. This indicates that the necessary membrane receptors required for their protective effects are present in both the apical and the basolateral membranes of Caco-2 cell monolayers. Probiotic protein, $\mathrm{p} 75$, also prevented hydrogen peroxide-induced disruption of barrier function in T84 and HT29 cell monolayers, indicating that this effect is not confined to one cell line.

Both $\mathrm{p} 40$ and $\mathrm{p} 75$ are novel bacterial proteins, which induce intestinal epithelial cell signal transduction and anti-apoptotic responses ${ }^{[5]}$. The deduced full-length $\mathrm{p} 40$ sequence is $79 \%$ identical to the sequence of a 396 amino acid protein of unknown function in Lactobacillus casei 334 (NCBI GeneBank COG3883) and the partial sequence of p75, which is most closely related to a 493amino-acid cell wall-associated hydrolase of L. casei 334 (NCBI GeneBank COG0791). The predicted molecular mass of the full-length cell wall-associated hydrolase of Lactobacillus casei $334(49 \mathrm{kDa})$ differs substantially from the molecular mass of the LGG $\mathrm{p} 75$ protein. The $\mathrm{p} 40$ gene sequence and the partial p75 gene sequence do not show significant similarity and the experimentally determined $\mathrm{N}$-terminal amino acid sequences of these two proteins are not related. Thus, based on the available sequence data, there is no evidence to suggest that $\mathrm{p} 40$ is a degradation product of p75. However, it is possible that there could be sequence similarity between p40 and the uncharacterized C-terminal portion of p75. The mechanisms of p40 and p75 regulating cell signaling are an area of active investigation including identification of potential interacting proteins and potential receptor(s), however no candidates have been clearly identified so far.

Both p40 and p75 effectively prevented hydrogen peroxide-induced loss of tight junction and adherens junction proteins from the detergent-insoluble fractions. Previous studies showed that the tight junction and adherens junction proteins, occludin, ZO-1, E-cadherin and $\beta$-catenin are associated with the F-actin-rich Triton-insoluble fractions in an intact epithelium and these fractions of the tight junction and adherens junction proteins correlate well with the integrity of tight junction ${ }^{[13]}$. Hydrogen peroxide caused a dissociation of these tight junction and adherens junction proteins from the detergent-insoluble fractions, suggesting that the dissociation of tight junction and adherens junction proteins from the actin cytoskeleton is one of the mechanisms involved in this tight junction disruption. Probiotic proteins somehow prevent hydrogen peroxide-induced loss of interaction between the actin cytoskeleton and the tight junction and adherens junction proteins. Therefore, the protective effects of P40 and P75 are likely to be mediated by specific cellular mechanisms. It was shown that $\mathrm{p} 40$ and p75 activate Akt in the intestinal epithelial cells ${ }^{[5]}$. However, phopho-Akt was undetectable in p40 or p75treated Caco- 2 cells. Therefore, probiotic-mediated Akt activation may play a role in cell survival effect, but is not involved in the protection of tight junction from hydrogen peroxide (data not shown).

Attenuation of the P40 and P75-mediated protection of barrier function and tight junction from hydrogen peroxide by Ro-32-0432 (a PKC-selective inhibitor) indicates that PKC activity is involved in the tight junction protection by probiotic secretory proteins. Ro-32-0432 is known to selectively inhibit the activities of $\mathrm{PKC} \alpha, \mathrm{PKC} \varepsilon$ and PKC $\beta \mathrm{I}$. Both p40 and p75 rapidly increase the membrane translocation of $\mathrm{PKC} \varepsilon$ and PKC $\beta \mathrm{I}$; membrane localization of PKC $\alpha$ was unaltered. This indicates that $\mathrm{p} 40$ and $\mathrm{p} 75$ induce the activation of PKCE and PKC $\beta I$ and this activation of the PKC isoforms may be required for the protection of tight junction from hydrogen peroxide. Maximal activation of PKC $\beta \mathrm{I}$ was achieved by $2 \mathrm{~min}$, while PKCE translocation was detectable only at $15 \mathrm{~min}$ after p75 administration. This suggests that PKC $\beta$ I activation may be one of the initial events in the mechanism of probiotic-mediated protection of tight junction and adherens junction. PKCE may play a role in the down stream events of the signaling pathway involved in this process.

Studies also showed that MAP kinase activity is involved in the p40 and p75-mediated prevention of the hydrogen peroxide-induced disruption of tight junction 
and increase in paracellular permeability. U0126 (a MEK selective inhibitor) attenuates the protective effect of p40 and p75 on tight junction, indicating that these probiotic proteins activate ERK1/2 via MEK activity. This is confirmed by the demonstration that both $\mathrm{p} 40$ and p75 rapidly increase the level of p-ERK in Caco-2 cells. A recent study demonstrated that activation of ERK plays a crucial role in the EGF-mediated prevention of hydrogen peroxide-induced disruption of tight junction and the increase in paracellular permeability ${ }^{[22]}$. Phospho-ERK directly interacted with occludin and prevented hydrogen peroxide-induced dephosphorylation of occludin on Thr residues. A similar mechanism may play a role in the probioticmediated protection of the tight junction from hydrogen peroxide-induced insult.

PKC inhibitors failed to prevent probiotic-induced activation of ERK1/2 and similarly, MEK inhibitor failed to prevent probiotic-induced membrane translocation of PKC $\varepsilon$ or PKC $\beta$ I. These data suggested that probiotic-induced activation of MAP kinase and PKC signaling pathways are independent of one another. Therefore, it is likely that probiotic proteins activate multiple signaling pathways and coordination these signaling pathways is required for the probioticmediated protection of intestinal epithelial tight junctions. The precise mechanism involved in PKC and MAP kinase activation in tight junction regulation is not known. A recent study suggested that PKC activation is involved in stabilization of perijunctional actomyosin ring $^{[21]}$. MAP kinase activation may regulate the Thr-phosphorylation of Occludin.

\section{CONCLUSION}

The evidence is convincing that probiotics protect the gastrointestinal mucosa from a variety of insults including infection by harmful bacteria. Several mechanisms appear to be associated with this mucosal protective role of probiotics. One such mechanism may involve preservation of the integrity of epithelial tight junctions and therefore the gut barrier function. Recent studies demonstrate that soluble proteins of Lactobacillus rhamnosus GG are effective in protecting the epithelia tight junctions from oxidative stress by activating intracellular signaling mechanisms. Therefore, probiotics and the soluble proteins may offer potential therapeutic benefits in the prevention and treatment of gastrointestinal diseases and possibly other systemic pathophysiological conditions.

\section{ACKNOLEDGEMENT}

This study was supported by NIH grants DK55532, AA12307, DK56008 and DK081134.

\section{REFERENCES}

1. Lilly, D.M. and R.H. Stillwell, 1965. Probiotics: Growth-promoting factors produced by microorganisms. Science, 147: 747-748. http://www.ncbi.nlm.nih.gov/entrez/query.fcgi?cm $\mathrm{d}=$ Retrieve $\& \mathrm{db}=$ PubMed\&dopt=Citation\&list_uids $=14242024$

2. Walker, W.A., 2006. Immunology. Curr. Opin. Gastroenterol., 22: 641-643. DOI: 10.1097/01.mog.0000245531.35518.77

3. Brown, A.C. and A. Valiere, 2004. Probiotics and medical nutrition therapy. Nutr. Clin. Care, 7: 56-68. http://www.ncbi.nlm.nih.gov/entrez/query.fcgi?cm $\mathrm{d}=$ Retrieve \&db=PubMed\&dopt=Citation\&list_uids $=15481739$

4. Resta-Lenert, S. and K.E. Barrett, 2006. Probiotics and commensals reverse TNF $\alpha$ and IFN $\gamma$-induced dysfunction in human intestinal epithelial cells. Gastroenterology, 130: 731-746. DOI: S00165085(05)02525-4

5. Yan, F. and D.B. Polk, 2002. Probiotic bacterium prevents cytokine-induced apoptosis in intestinal epithelial cells. J. Biol. Chem., 277: 50959-50965. DOI: $10.1074 /$ jbc.M207050200

6. Yan, F., H. Cao, T.L. Cover, R. Whitehead, M.K. Washington and D.B. Polk, 2007. Soluble proteins produced by probiotic bacteria regulate intestinal cell survival and growth. Gastroenterology, 132: 562-75. DOI: 10.1053/j.gastro.2006.11.022

7. Yan, F. and D.B. Polk, 2006. Probiotics as functional food in the treatment of diarrhea. Curr. Opin. Nutr. Metab. Care, 9: 717-721. DOI: 10.1097/01.mco.0000247477.02650.51

8. Gorbach, S.L., T.W. Chang and B. Goldin, 1987. Successful treatment of relapsing Clostridium difficile colitis with Lactobacillus GG. Lancet, 2: 1519-1519.

http://www.ncbi.nlm.nih.gov/entrez/query.fcgi?cm $\mathrm{d}=$ Retrieve $\& \mathrm{db}=$ PubMed\&dopt=Citation\&list_uids $=2892070$

9. Anderson, J.M. and C.M. Van Italie, 1995. Tight junctions and the molecular basis for regulation of paracellular permeability. Am. J. Physiol., 269: G467-G475.

http://www.ncbi.nlm.nih.gov/entrez/query.fcgi?cm $\mathrm{d}=$ Retrieve $\& \mathrm{db}=$ PubMed\&dopt=Citation\&list_uids $=7485497$ 
10. Rao, R.K., 2008. The mechanisms of oxidative stress-induced disruption of epithelial and endothelial tight junctions. Fron. Biosci., 13: 7219-7226.

11. Basuroy, S., P. Sheth, D. Kuppuswamy, S. Balasubramanian, R.M. Ray and R.K. Rao, 2003. Expression of kinase-inactive c-Src delays oxidative stress-induced disassembly and accelerates calcium-mediated reassembly of tight junctions in the Caco-2 cell monolayer. J. Biol. Chem., 278: 11916-11924. DOI: 10.1074/jbc.M211710200

12. Rao, R.K., R.D. Baker, S.S. Baker, A. Gupta and M. Holycross, 1997. Oxidant-induced disruption of intestinal epithelial barrier function: Role of protein tyrosine phosphorylation. Am. J. Physiol., 273: G812-G823.

http://www.ncbi.nlm.nih.gov/entrez/query.fcgi?cm $\mathrm{d}=$ Retrieve $\& \mathrm{db}=$ PubMed\&dopt=Citation\&list_uids $=9357822$

13. Rao, R.K., S. Basuroy, V.U. Rao, K.J. Karnaky and A. Gupta, 2002. Tyrosine phosphorylation and dissociation of E-cadherin-beta-catenin complexes from the cytoskeleton by oxidative stress. Biochem. J., 368: 471-481. DOI: 10.1042/BJ20011804

14. Rao, R.K., L. Li, S.S. Baker, R.D. Baker and A. Gupta A, 2000. Glutathione oxidation and inhibition of protein tyrosine phosphatase in hydrogen peroxide-induced increase in paracellular permeability. Am. J. Physiol., 279: G332-G340. http://www.ncbi.nlm.nih.gov/entrez/query.fcgi?cm $\mathrm{d}=$ Retrieve $\& \mathrm{db}=$ PubMed\&dopt $=$ Citation\&list_uids $=10915642$

15. Sheth, P., S. Basuroy, C. Li, A.P. Naren and R.K. Rao, 2003. Role of phosphatidylinositol 3kinase in oxidative stress-induced disruption of tight junctions. J. Biol. Chem., 278: 49239-49245. DOI: $10.1074 /$ jbc.M305654200

16. Clayburgh, D.R., M.W. Musch, M. Leitgs, Y.X. Fu and J.R. Turner, 2006. Coordinated epithelial NHE3 inhibition and barrier dysfunction are required for TNF-mediated diarrhea in vivo. J. Clin. Investment, 116: 2682-94. DOI: 10.1172/JCI29218
17. Utech, M., A.I. Ivanov, S.N. Samarin, M. Bruewer and J.R. Turner et al., 2005. Mechanism of IFNgamma-induced endocytosis of tight junction proteins: Myosin II-dependent vacuolarization of the apical plasma membrane. Mol. Biol. Cell, 16: 5040-52. DOI: 10.1091/mbc.E05-03-0193

18. Shifflett, D.E., D.R. Clayburgh, A. Koutsouris, J.R. Turner and G.A. Hecht, 2005. Enteropathogenic E. coli disrupts tight junction barrier function and structure in vivo. Lab. Invest., 85: 1308-24. DOI: 10.1038/labinvest.3700330

19. Ukena, S.N., A. Singh, U. Dringenberg, R. Engelhardt and U. Seidler et al., 2007. Probiotic Escherichia coli Nissle 1917 inhibits leaky gut by enhancing mucosal integrity. PLoS ONE., 12: 1-9. DOI: 10.1371/journal.pone.0001308

20. Seth, A., F. Yan, D.B. Polk and R.K. Rao, 2008. Probiotics Ameliorate the hydrogen peroxideinduced epithelial barrier disruption by a PKC and MAP kinase-dependent mechanism. Am. J. Physiol., 294: G1016-G1069. DOI: 10.1152/ajpgi.00202.2007

21. Suzuki, T., A. Seth and R.K. Rao, 2008. Role of PLC $\gamma$-mediated activation of PKC $\varepsilon$ and PKC $\beta \mathrm{I}$ in EGF-induced protection of tight junctions from acetaldehyde in Caco-2 cell monolayers. J. Biol. Chem., 283: 3574-3583. DOI: 10.1074/jbc.M709141200

22. Basuroy, S., A. Seth, B. Elias, A.P. Naren and R.K. Rao, 2006. MAP kinase interacts with occludin and mediates EGF-induced protection of tight junctions from hydrogen peroxide. Biochem. J., 393: 69-77. DOI: 10.1042/BJ20050959 